\title{
Pandemic dentistry: a call for reducing dental aerosols?
}

A clinical study measuring dental aerosols with and without a HVE device. Br Dent J 2020; 229: http://dx.doi.org/10.1038/s41415-020-2274-3.

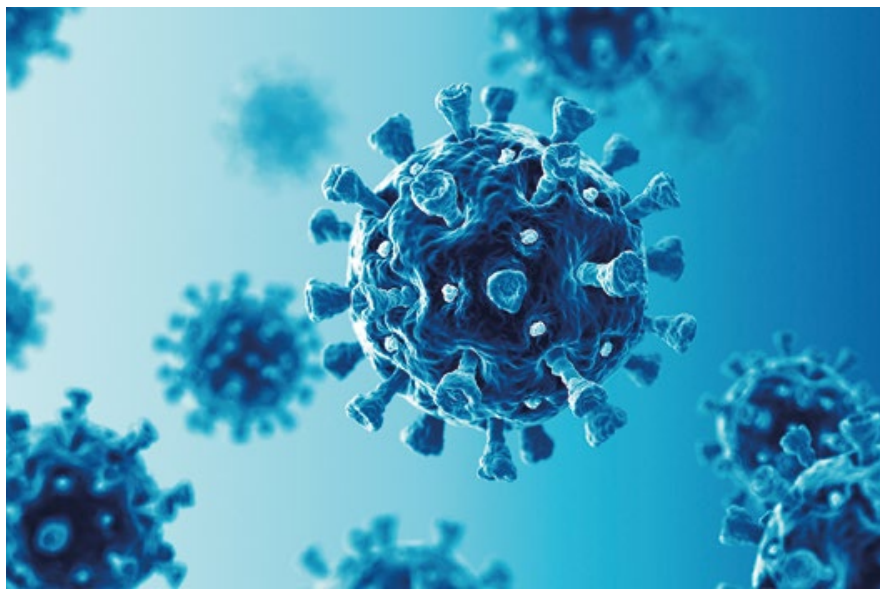

As the COVID-19 crisis continues, the mechanism in which the SARS-CoV-2 virus is spread remains under investigation. The current understanding that the virus is transmitted through aerosol droplets has severely restricted previously routine dental procedures with governments and health authorities recommending only emergency and essential treatments go ahead. Dental aerosol producing procedures emit droplets of varying sizes. Larger particles can settle up to two metres away on surrounding surfaces whereas smaller particles can remain in the air for minutes or even hours, potentially causing respiratory infections if inhaled.

This paper aims to compare the particle count measured from dental aerosol procedures with and without the use of a high-volume extraction (HVE) device.

Four clinicians performed five aerosol generating restorative procedures on a phantom head and repeated the same five procedures with the addition of a HVE device positioned in front of the patient's mouth. An industrial Trotec PC220 particle counter was positioned at the average recorded working distance of the clinicians. The particle counter recorded the number of PM1 (particles sized 0.1 or less microns), PM2.5 (particles sized 1-2.5 microns) and PM10 particulate (particles sized $2.5-10$ microns) produced in each procedure.

The study found that when any dental procedures were undertaken, production of PM1 particulate remained within control measurements taken before the procedures. Conversely, both the number of PM2.5 and PM10 particulates increased to approximately two to three times background levels during all the aerosol procedures when no HVE device was in use. When an external HVE device was then introduced, the aerosol particle count was decreased with every dental procedure by around two dozen $\mu \mathrm{m}$ per cubic metre. This excludes, however, the reduction in PM1 particulate generated in the 3-in-1 procedures, as the reduction was not statistically significant.

The study is limited with its relevance to SARS-CoV-2 as investigations into how the virus is transmitted are still ongoing. Yet, if it is considered that the SARS-CoV-2 virus could be contained within aerosols and particulate, the study results show a possible heightened risk of transmission from dental aerosol procedures that generate a significant increase in PM2.5 and PM10 particulate.

The dental profession must explore options to mitigate the risk of virus transmission during the pandemic. Worldwide, minimum protection has included high risk personal protective equipment with the UK also advising a fallow time after aerosol procedures. The authors of this paper call for an expanded study to consider the effect of certain risk mitigating factors.

By Laura Cook, Dental Student, University of Leeds, UK

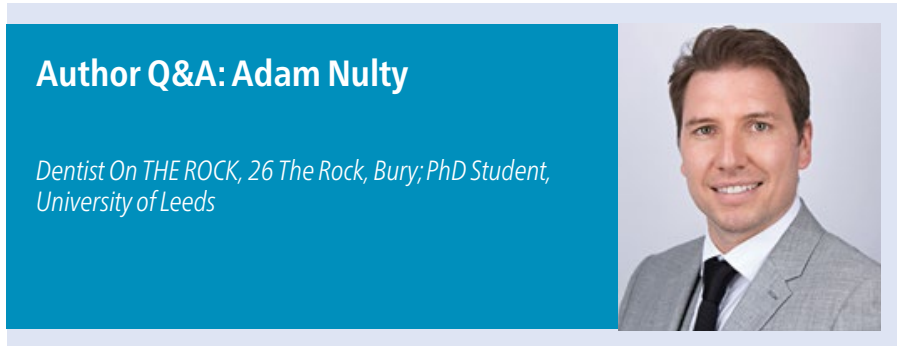

Why did you decide to undertake this study?

We decided to undertake this study to benefit dental practices nationally and indeed internationally. The SARS-CoV-2 pandemic has provided a great many difficulties in general dental practice due to the uncertainty of both risk and the provision of care. When working with the IDDA, Pandora, IPO and the FGDP (with the BAPD) in the provision of various research backgrounds and standard operating procedures etc, it became clear that political issues meant that new sound research on unknown factors would be critical to enable the use of recent technologies and justify their use in mitigating risk of aerosols with the overall aim to benefit the dental treatment of patients.

\section{Did any of the results surprise you?}

At the inception of this study, it quickly became apparent that the use of an external HVE was reducing the aerosol particle count. However, on examination and statistical analysis of the results, it was surprising to see just how much of an effect there would be.

What do you think the next steps should be considering your findings? We are currently in the process of further expanding this study to assess the impact of increased aerosol generation duration, as well as the cumulative effect of other risk mitigating factors. We would also like to look at how much of an impact these devices have on expiratory events such as coughing or sneezing directly and at various distances. If further study shows that aerosol particulate is also reduced in an in vivo clinical setting, it may be possible to use HVE external extraction devices to effectively reduce and mitigate the associated risk with aerosol procedures. We would like to see the FGDP use these results to incorporate into their advice on fallow time. 